A joint survival-longitudinal modelling approach for the dynamic prediction of rehospitalization in telemonitored chronic heart failure patients. Supplementary material

NJAGI, Edmund; Rizopoulos, Dimitri; MOLENBERGHS, Geert; DENDALE, Paul \& WILLEKENS, Koen (2013) A joint survival-longitudinal modelling approach for the dynamic prediction of rehospitalization in telemonitored chronic heart failure patients.. In: Statistical modelling, 13 (3), p. 179-198.

DOI: $10.1177 / 1471082 \times 13478880$

Handle: http://hdl.handle.net/1942/16470 


\section{A Joint Survival-Longitudinal Modelling Approach for the Dynamic Prediction of Rehospitalization in Telemonitored Chronic Heart Failure Patients}

\section{Web Appendix}

\section{Edmund Njeru Njagi*}

Research Assistant, I-BioStat, Universiteit Hasselt, Diepenbeek, Belgium

Dimitris Rizopoulos

Professor, Department of Biostatistics, Erasmus University Medical Center,

Rotterdam, the Netherlands

\section{Geert Molenberghs}

Professor, I-BioStat, Universiteit Hasselt \& KU Leuven, Diepenbeek \& Leuven, Belgium

\section{Paul Dendale}

Professor, Jessa Hospital, Heart Centre Hasselt, Hasselt, Belgium

\& Universiteit Hasselt, Faculty of Medicine and Life Sciences, Diepenbeek, Belgium

\section{Koen Willekens}

Medical Student, Katholieke Universiteit Leuven, Faculty of Medicine, B-3000 Leuven, Belgium

*Address for correspondence: I-BioStat, Universiteit Hasselt, Agoralaan 1, B-3590 Diepenbeek, Belgium. Email: edmund.njagi@uhasselt.be 
Table A.1: Systolic Blood Pressure. DDIs.

\begin{tabular}{|c|c|c|}
\hline & Time window $\Delta t$ & DDI \\
\hline \multirow[t]{4}{*}{ First Step Model } & 2 & 0.6009 \\
\hline & 4 & 0.6048 \\
\hline & 8 & 0.6760 \\
\hline & 16 & 0.6145 \\
\hline \multicolumn{3}{|c|}{ Second Step Model } \\
\hline \multirow[t]{4}{*}{ NTproBNP } & 2 & 0.4917 \\
\hline & 4 & 0.4995 \\
\hline & 8 & 0.5712 \\
\hline & 16 & 0.5223 \\
\hline \multirow[t]{4}{*}{ Heart Rhythm } & 2 & 0.5844 \\
\hline & 4 & 0.6038 \\
\hline & 8 & 0.6551 \\
\hline & 16 & 0.6064 \\
\hline \multirow[t]{4}{*}{ NYHA } & 2 & 0.5585 \\
\hline & 4 & 0.5585 \\
\hline & 8 & 0.6561 \\
\hline & 16 & 0.5869 \\
\hline \multirow[t]{4}{*}{ Sex } & 2 & 0.5977 \\
\hline & 4 & 0.6051 \\
\hline & 8 & 0.6597 \\
\hline & 16 & 0.6304 \\
\hline \multirow[t]{4}{*}{ LVEF } & 2 & 0.4823 \\
\hline & 4 & 0.4863 \\
\hline & 8 & 0.5839 \\
\hline & 16 & 0.5749 \\
\hline \multirow[t]{4}{*}{ Age } & 2 & 0.6453 \\
\hline & 4 & 0.6453 \\
\hline & 8 & 0.6771 \\
\hline & 16 & 0.6748 \\
\hline
\end{tabular}


Table A.2: Weight. DDls.

\begin{tabular}{|c|c|c|}
\hline & Time window $\Delta t$ & DDI \\
\hline \multirow[t]{4}{*}{ First Step Model } & 2 & 0.3877 \\
\hline & 4 & 0.3877 \\
\hline & 8 & 0.4648 \\
\hline & 16 & 0.5020 \\
\hline \multicolumn{3}{|c|}{ Second Step Model } \\
\hline \multirow[t]{4}{*}{ NTproBNP } & 2 & 0.5885 \\
\hline & 4 & 0.5885 \\
\hline & 8 & 0.6392 \\
\hline & 16 & 0.5388 \\
\hline \multirow[t]{4}{*}{ Heart Rhythm } & 2 & 0.5433 \\
\hline & 4 & 0.5515 \\
\hline & 8 & 0.5747 \\
\hline & 16 & 0.5199 \\
\hline \multirow[t]{4}{*}{ NYHA } & 2 & 0.5227 \\
\hline & 4 & 0.5304 \\
\hline & 8 & 0.5598 \\
\hline & 16 & 0.5044 \\
\hline \multirow[t]{4}{*}{ Sex } & 2 & 0.5317 \\
\hline & 4 & 0.5317 \\
\hline & 8 & 0.5434 \\
\hline & 16 & 0.5047 \\
\hline \multirow[t]{4}{*}{ LVEF } & 2 & 0.4565 \\
\hline & 4 & 0.4605 \\
\hline & 8 & 0.5085 \\
\hline & 16 & 0.4685 \\
\hline \multirow[t]{4}{*}{ Age } & 2 & 0.6634 \\
\hline & 4 & 0.6634 \\
\hline & 8 & 0.6434 \\
\hline & 16 & 0.5978 \\
\hline
\end{tabular}

